\title{
In vitro studies with a pulsed neodymium/YAG laser
}

\author{
S VENKATESH,' S GUTHRIE, ' W S FOULDS, ' W R LEE,' \\ F R CRUICKSHANK, ${ }^{2}$ AND R T BAILEY ${ }^{2}$ \\ From the 'Tennent Institute of Ophthalmology, University of Glasgow; and the ${ }^{2}$ Department of Pure \\ and Applied Chemistry, University of Strathclyde, Glasgow
}

SUMmaRY The relationships between the destructive effects of Q-switched Nd/YAG laser pulses and a number of experimental parameters were studied for various target materials including in particular excised, fixed samples of human trabecular meshwork. The laser parameters altered were the pulse energy, the convergence angle of the focused beam, and the position of the focus of the beam relative to the target's axial position. The main finding was that it was possible to make deep holes, of a diameter less than $100 \mu \mathrm{m}$, in virtually transparent samples of trabecular meshwork with a laser delivery system of $6^{\circ}$ convergence and pulse energies of $14 \mathrm{~mJ}$ or more. The relevance of this and the other experimental results to the development of a reliable system for performing internal trabeculotomies for the treatment of open-angle glaucoma is presented.

Over the past few years interest has grown in the possible applications of high power pulsed neodymium/YAG lasers in ophthalmology, particularly in the destruction of transparent tissues. Modelocked Nd/YAG lasers, which give extremely high power and low energy pulses, have been used successfully clinically to open the anterior lens capsule before extracapsular extraction, to pierce secondary membranes, to treat pupillary block and updrawn pupil, and to cut vitreous bands. ${ }^{2} \mathrm{Q}$-switched neodymium/YAG lasers, which give higher pulse energies but lower peak powers, have been used experimentally on tissues of the anterior chamber, ${ }^{3}$ and more recently clinical success had been reported in a range of treatments-iridectomy, membranotomy in pseudophakia, membranotomy and dissection of vitreous bands, ${ }^{4}$ coreplasty, and the division of synechiae. ${ }^{5}$

Our long term objective is to develop a system based on a Q-switched Nd/YAG laser to perform internal trabeculotomies as a possible treatment for open-angle glaucoma. Previous attempts by others to use Q-switched pulses to treat open-angle glaucoma have not been successful possibly because of the collapse of Schlemm's canal around the site of the laser hole. ${ }^{6}$ Cases where new channels into the suprachoroidal space have been made have also failed to produce a lasting reduction in intraocular

Correspondence to Dr S Venkatesh, Tennent Institute of Ophthalmology, University of Glasgow, Glasgow G11 6NT. pressure. ${ }^{7}$ We would hope to avoid the risk of inducing Schlemm's canal to collapse by making laser holes of very small diameter, say $50 \mu \mathrm{m}$, spaced around the band, each penetrating the meshwork through to the canal. The demands of such a technique on precision of focusing, reproducibility of the laser output, and confidence in its predicted effect would be great, and, as a first step towards our objective, a series of systematic studies was carried out to investigate the relationships between the observed destructive effects of Q-switched pulses and experimental parameters such as pulse energy, convergence angle of the focused beam, and the nature of the target.

\section{Materials and methods}

The laser system used in these studies was the HyperYAG 2000 produced by JK Lasers Ltd. It was operated at its fundamental wavelength of $1.06 \mu \mathrm{m}$. Pulse durations were compressed to between 30 and 40 ns by Q-switching, so that high powers could be achieved with relatively low energies. A fast photodiode detector (Centronic APD 05 4R) was used to check the pulse duration occasionally. One of its typical output traces, proportional to pulse power versus time, is shown in Fig. 1.

The laser pumping voltage could be altered to vary the pulse energy. The actual pulse energy output was measured before and after each experiment with a photodiode based energy monitor (JK Lasers Ltd). In these experiments the pulse energies used were in 

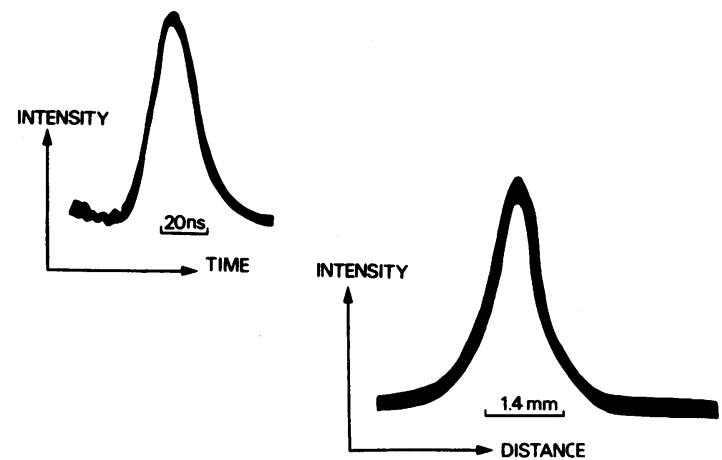

Fig. 1 Temporal and spatial intensity profiles of $Q$-switched Nd/YAG pulses.

the range from $3 \mathrm{~mJ}$ to $30 \mathrm{~mJ}$ corresponding to pulse powers in the range from $10^{5}$ to $10^{6} \mathrm{~W}$.

The laser output was carefully channelled into its fundamental transverse mode, which offered two advantages - the theoretical limit for the focused beam diameter is smaller than for other modes, and the variability in the pattern of intensity from one pulse to another is minimised. The mode pattern of the unfocused Nd/YAG beam was measured periodically with a photodiode array detector (EG and G Reticon RL 512G), and adjustments were made to the laser cavity optics if necessary to regain the fundamental mode. Fig. 1 shows a typical output trace proportional to pulse power versus distance. From such spatial intensity profiles the beam diameter was found to be $1.5 \mathrm{~mm}$. (The diameter is measured across the beam at the points where the signal intensity is $1 / \mathrm{e}$ (that is, $1 / 2 \cdot 718$ ) of its peak value.)

Fig. 2 shows the experimental arrangement used for these studies. A low power continuous wave helium neon laser beam was directed coaxially into the invisible $\mathrm{Nd} / \mathrm{YAG}$ beam to mark its path and allow visual focusing. The delivery system supplied with the laser was removed, and the two beams were delivered to the target through a beam expanding telescope and a single focusing lens. Different lenses could be used in this position to converge the Nd/YAG beam at different angles.
The target was mounted on a micropositioning stage, so that its position along the axis of the beams could be altered and set with precision relative to the point at which the HeNe beam could be seen to be focused. The viewing system used to observe this focusing, and so position the target, was quite separate from the laser focusing optics and consisted of a binocular microscope with fibre optic illumination.

Glutaraldehyde-fixed human tissue, obtained at necropsy was used in this study. As the eventual objective was to make holes through the outflow system, many of the experiments used excised pieces of anterior chamber angle tissue with the uveal meshwork surface facing the $\mathrm{Nd} / \mathrm{YAG}$ beam. In some cases a suspension of saccharated iron oxide was injected into Schlemm's canal in an attempt to mimic the in vivo situation when blood might be present in the canal and, if so, might affect the tissue response to the laser radiation. Corneal tissue was also used as a target, because in the clinical application of this work the converging Nd/YAG beam would pass through the cornea on its way to the meshwork. It was therefore necessary to know whether the cornea would be likely to suffer damage when situated only a few mm away from the focal spot of the Nd/YAG beam.

A smooth, transparent plastic material (Mylar) was also chosen to act as a relatively homogeneous and reproducible non-biological target.

The morphology of the destructive effects of the $\mathrm{Nd} /$ YAG laser pulses on the various targets was studied primarily by scanning electron microscopy (Jeol Ltd JSM-T200), for which the tissue samples were prepared by critical point drying. Image analysis (Micro Measurements Ltd, Optomax) was carried out on the scanning electron micrographs to give measurements of hole areas, and a stereoscope with a parallax measurement unit (Cartographic Engineering Ltd) was used on tilted pairs of micrographs to give hole depth measurements. Some of the scanned tissue specimens were reprocessed for Araldite embedding by a technique to be described elsewhere, and $1.0 \mu \mathrm{m}$ toluidine blue stained sections were

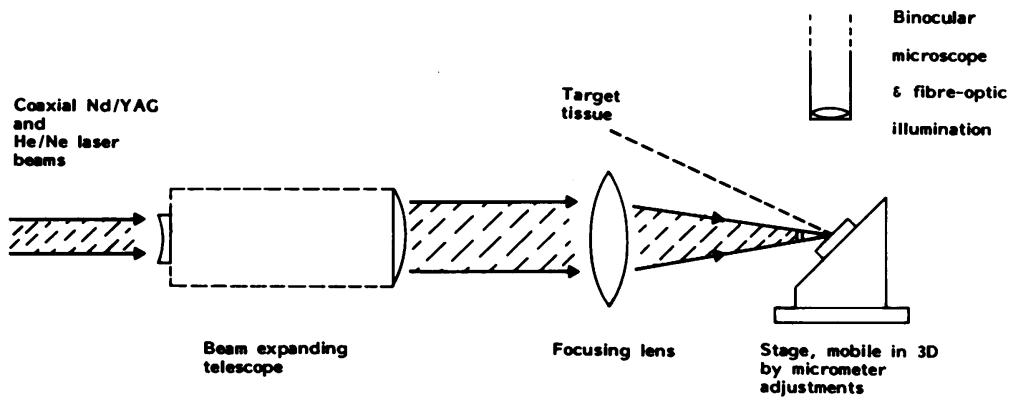

Fig. 2 Schematic diagram of experimental delivery system. 
Fig. 3 Laserhole made by a single $30 \mathrm{~mJ}$ pulse converging at $2^{\circ}$ on to tissue positioned at the HeNe focus. $M=$ Uveal meshwork; $R=$ Red blood cells.

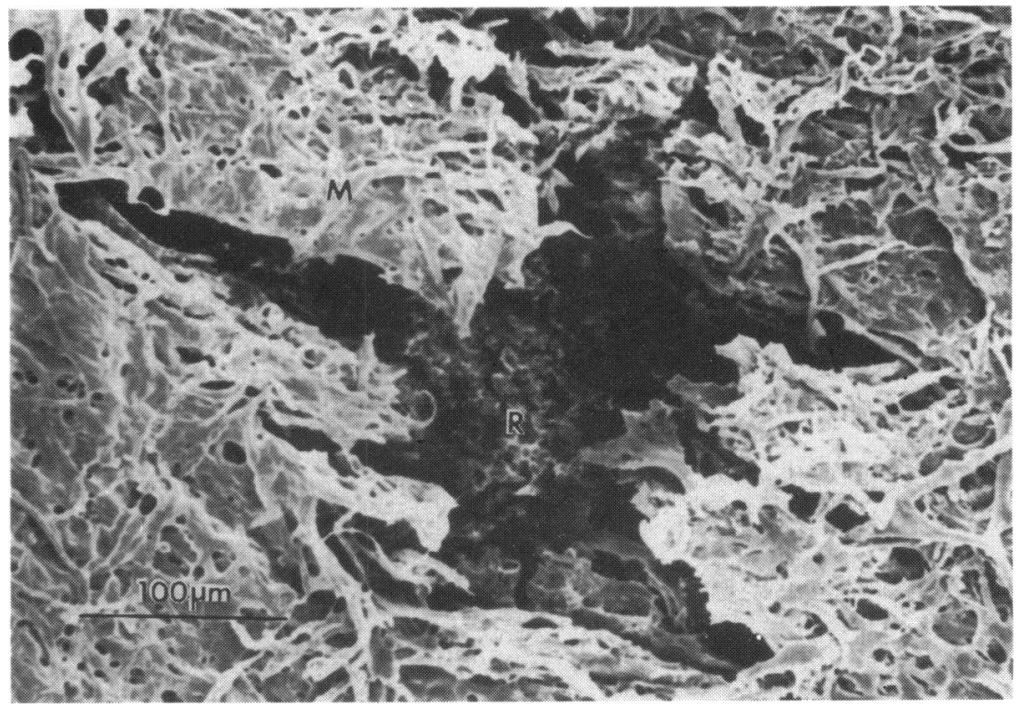

studied by light microscopy (Leitz Dialux 20/Wild Photoautomat MPS 45).

\section{Procedure and results}

Six sequential sets of experiments were carried out as follows:

Meshwork tissue at low convergence angle. A lens of focal length $52 \mathrm{~mm}$ was used to focus the $\mathrm{Nd} / \mathrm{YAG}$ beam on to the uveal surface of meshwork samples. This would give a beam convergence angle of only $2^{\circ}$, much lower than we expected to use in our in vivo application, but it did offer a long working distance, which was very useful in this preliminary work. Single pulses of $30 \mathrm{~mJ}$ each were fired into tissue positioned at the point of focus of the HeNe beam. Determining this focal point with a precision of even $100 \mu \mathrm{m}$ was found to be difficult.

Scanning electron microscopy of the tissue samples subsequently showed well defined holes at 13 out of 22 target sites. Holes that were clearly seen to penetrate through to Schlemm's canal were made in five cases. Fig. 3 shows one of these holes.

Measurements of hole area and depth yielded coefficients of variation of over $70 \%$ indicating great variability in the dimensions of holes made in trabecular tissue under apparently identical conditions. In nine cases no hole was made at all. A simpler target material was therefore chosen for the next set of experiments.

Mylar at low convergence angle. Single pulses of 10 $\mathrm{mJ}$ were fired into plastic placed at a series of positions between $0.5 \mathrm{~mm}$ in front of the $\mathrm{HeNe}$ focus and 3.5 $\mathrm{mm}$ beyond it. The plastic was then coated with gold and observed by scanning electron microscopy.

Large variations were again found in hole area and depth at a given target position shown by coefficients of variation of approximately $50 \%$. Possibly because

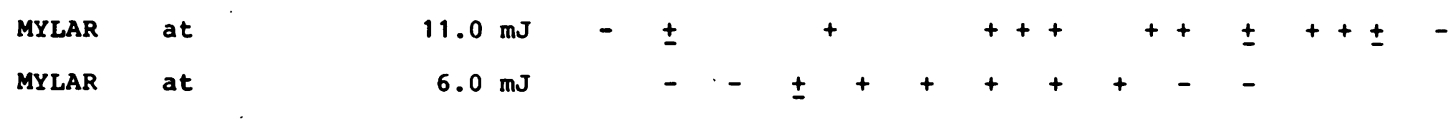

CORNEAL ENDOTHELIUM at $30.0 \mathrm{~mJ}$
CORNEAL ENDOTHELIUM at $13.0 \mathrm{~mJ}$
CORNEAL EPITHELIUM at $11.0 \mathrm{~mJ}$

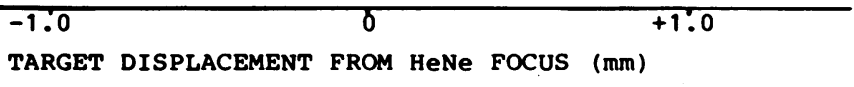

Fig. 4 Effects of Nd/YAG pulses at a convergence angle of $6^{\circ}$ targeted on Mylar and human cornea. $+=$ Hole made; $-=N o$ hole made. 


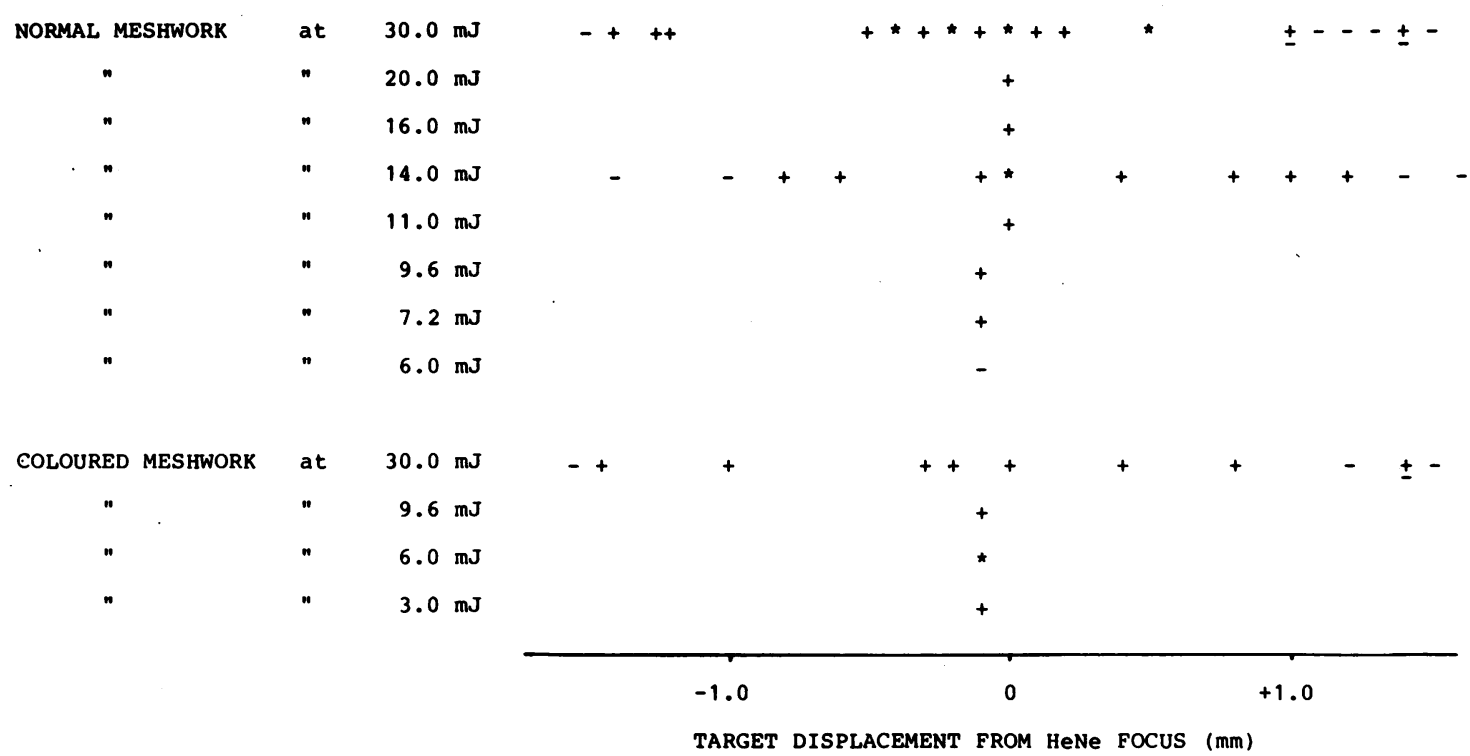

Fig. 5 Effects of Nd/YAG pulses at a convergence angle of $6^{\circ}$ targeted on normal and artificially coloured human meshwork. $+=$ Hole made $;{ }^{*}=$ Hole extending through to Schlemm's canal made $;-=$ No hole made.

of this no systematic change in area or depth with target position was observed. The main finding was that all 75 target sites fired at did show laser damage, though the target position had varied over a range of $4 \mathrm{~mm}$. The effective depth of focus of this delivery system was at least $4 \mathrm{~mm}$ then, obviously too large for the intended clinical application. The focusing lens was therefore replaced with one of shorter focal length for the next set of experiments.

Mylar at high convergence angle. A lens of focal length $14.6 \mathrm{~mm}$ was used to focus $\mathrm{Nd} / \mathrm{YAG}$ pulses at a convergence angle of $6^{\circ}$ on to pieces of Mylar held at a series of positions between $1.2 \mathrm{~mm}$ in front of the HeNe focus and $1.4 \mathrm{~mm}$ beyond it. Single pulses of either $6 \mathrm{~mJ}$ or $11 \mathrm{~mJ}$ were used.

The results are indicated in the upper section of Fig. 4. They show that $11 \mathrm{~mJ}$ made holes over a target position range of approximately $2 \mathrm{~mm}$, so the depth of focus of the delivery system had indeed been reduced. It can also be seen that the depth of focus was reduced from $2 \mathrm{~mm}$ to approximately $1 \mathrm{~mm}$ by lowering the pulse energy to $6 \mathrm{~mJ}$. While carrying out

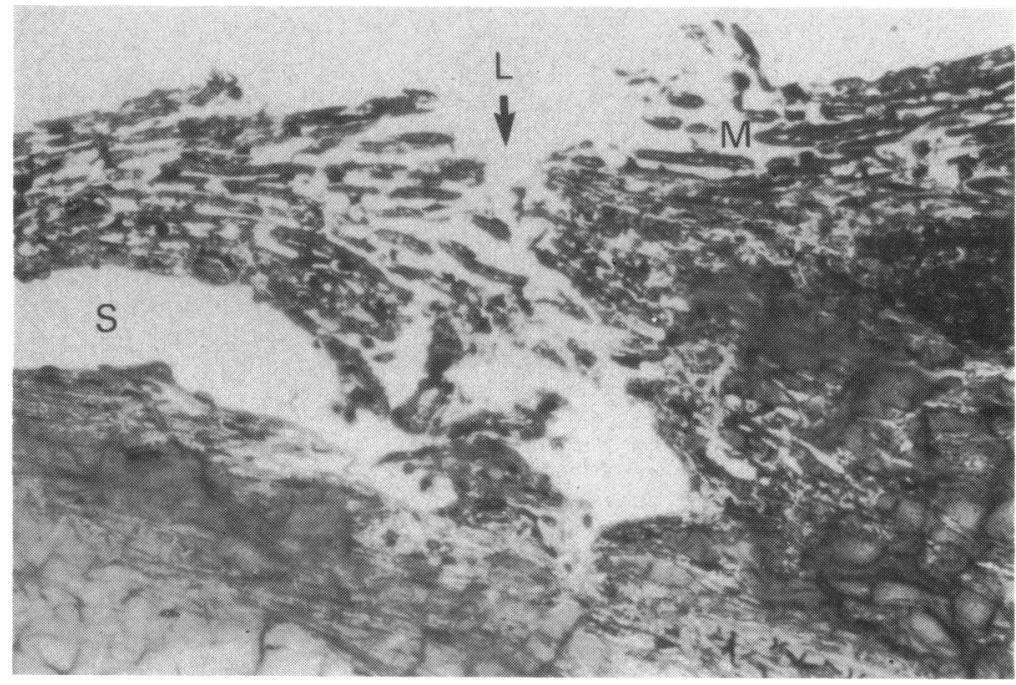

Fig. 6 A toluidine blue stained lum section of tissue previously positioned $0.5 \mathrm{~mm}$ beyond the HeNe focus and exposed to a single $30 \mathrm{~mJ}$ pulse at $6^{\circ}$ convergence. $S=$ Schlemm's canal; $L=$ Laser hole; $M=$ Uveal meshwork. ( $\times 298)$. 
this set of pulse firings we found it possible to locate the $\mathrm{HeNe}$ focus with a precision of approximately 10 $\mu \mathrm{m}$ with this high convergence system. These results were encouraging enough to warrant tissue experiments with the same system.

Cornea at high convergence angle. $\mathrm{Nd} / \mathrm{YAG}$ pulses, converged at an angle of $6^{\circ}$, were fired into corneal tissue held at positions between $300 \mu \mathrm{m}$ in front of the $\mathrm{HeNe}$ focus and $400 \mu \mathrm{m}$ beyond it. Single pulses of 30 $\mathrm{mJ}, 13 \mathrm{~mJ}$, or $11 \mathrm{~mJ}$ were fired into each target site. In some cases the corneal tissue was positioned with its epithelial surface facing the $\mathrm{Nd} / \mathrm{YAG}$ beam. In others the endothelial surface was exposed instead.

The results are indicated in the lower section of Fig. 4. It was found that even at $30 \mathrm{~mJ}, \mathrm{Nd} / \mathrm{YAG}$ pulses caused damage only if the corneal surface was positioned between $100 \mu \mathrm{m}$ in front of the $\mathrm{HeNe}$ focus and approximately $300 \mu \mathrm{m}$ beyond it. Such a small depth of focus for damage in the cornea suggested that the $6^{\circ}$ convergence angle system would be acceptable in that context for our eventual in vivo application.

Normal meshwork at high convergence angle. Excised samples of meshwork were held at a series of positions between $1.5 \mathrm{~mm}$ in front of the $\mathrm{HeNe}$ focus and $1.6 \mathrm{~mm}$ beyond it. Eight different Nd/YAG pulse energies in the range $6 \mathrm{~mJ}$ to $30 \mathrm{~mJ}$ were tried, firing a single pulse into each target site as before.

The results are indicated in the upper section of Fig. 5. There were four main points of interest in
Figs. 7A and B Scanning electron micrographs of tissue exposed to $N d / Y A G$ pulses at $6^{\circ}$ convergence.

A: Effect of one $30 \mathrm{~mJ}$ pulse fired into tissue position at $0.2 \mathrm{~mm}$ beyond the HeNe focus. $M=$ Uveal meshwork.

Fig. 7B: Effects of two $14 \mathrm{~mJ}$ pulses fired side by side into tissue positioned first at the $\mathrm{HeNe}$ focus then $0.4 \mathrm{~mm}$ beyond it. $M=$ Uveal meshwork; $S=S$ Shlemm's canal; $C=$ Collector channel .
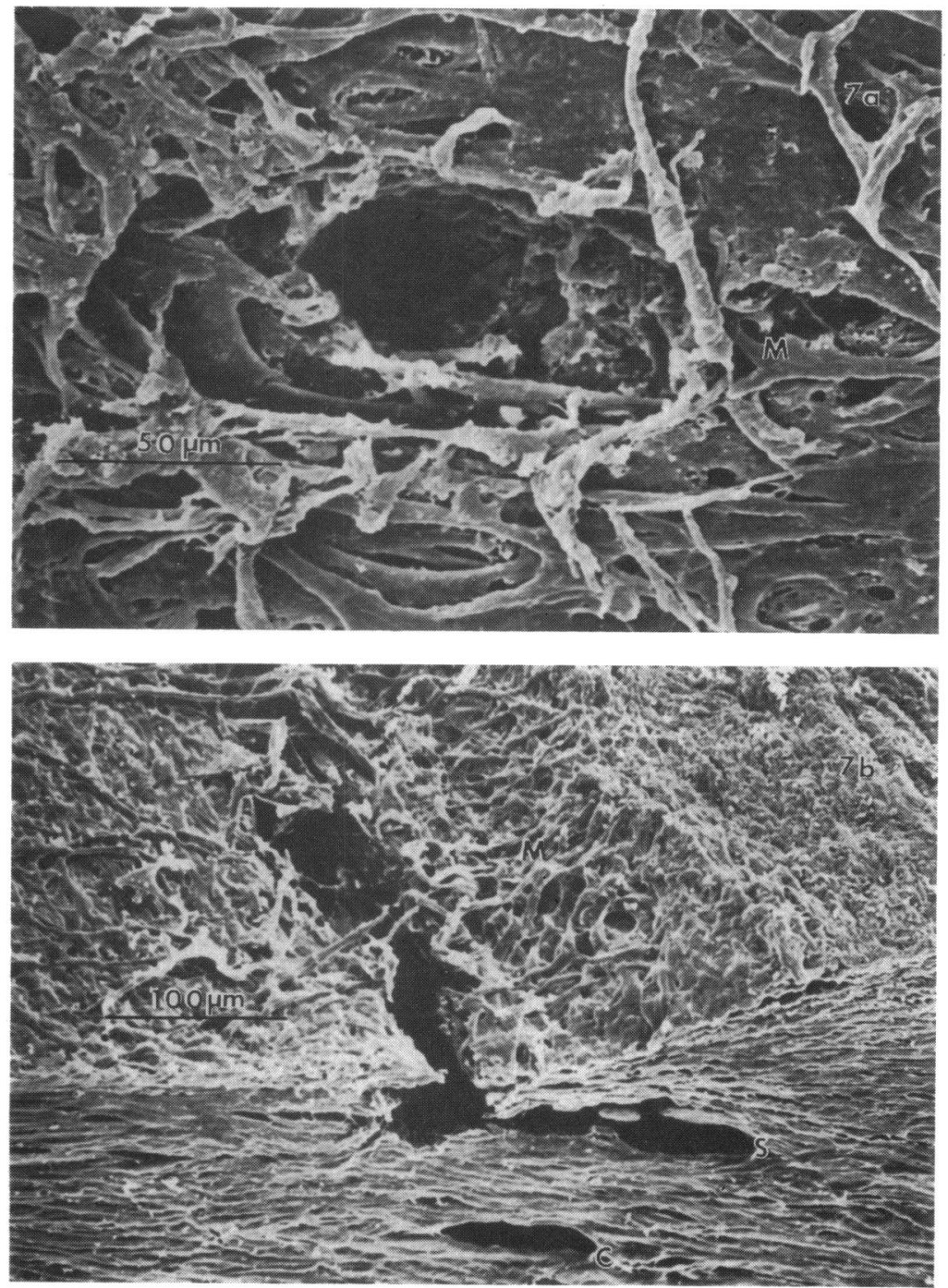
them. The effective depth of focus for making holes in meshwork with $30 \mathrm{~mJ}$ pulses was approximately $2.4 \mathrm{~mm}$ centred at the point of focus for the $\mathrm{HeNe}$ beam. This depth of focus was reduced as pulse energy was lowered. The threshold for meshwork damage was about $7 \mathrm{~mJ}$. The deeper holes were made when the target was closer to the $\mathrm{HeNe}$ focal point.

It was found that holes as small as $50 \mu \mathrm{m}$ in diameter but extending into Schlemm's canal could be made. Fig. 6 is a photomicrograph of a toluidine blue stained section cut through one of the laser holes. Fig. 7 shows scanning electron micrographs of some others.

Stained meshwork at high convergence angle. Four values of pulse energy in the range $3 \mathrm{~mJ}$ to $30 \mathrm{~mJ}$ were tried with the artificially coloured meshwork samples. In other respects the experimental protocol was as for the previous set of experiments.

The results are shown in the lower section of Fig. 5. It was found that $30 \mathrm{~mJ}$ pulses made holes over approximately the same range as for unstained tissue, but the threshold of damage was much lower. Even the lowest Q-switched pulse energy available with our system, $3 \mathrm{~mJ}$, resulted in a hole, and one pulse of only $6 \mathrm{~mJ}$ made a hole through to Schlemm's canal.

These results can be explained as follows. Laser damage by Q-switched pulses can occur by non-linear processes of fundamental structural breakdown, ${ }^{8}$ but only at very high power densities, of the order of $10^{10}$ watt $/ \mathrm{cm}^{2}$. These processes would be expected to apply to transparent and coloured matter equally, and our results at a pulse energy of $30 \mathrm{~mJ}$ confirm this. At lower power densities damage can occur by normal, linear absorption processes. The effectiveness of these processes would be expected to depend on the properties of the target matter, in particular its colour. Our results below $7 \mathrm{~mJ}$ fit this reasoning.

\section{Discussion}

This series of in vitro experiments has yielded information of interest and value in furthering our development of a noedymium/YAG laser system for performing internal trabeculotomies. The initial work, done with a small YAG convergence angle, showed the feasibility of making discrete holes through meshwork but also showed problems of poor reproducibility of the effects in terms of the dimensions of the holes made. This variability was found to depend partly on the irregularity of the tissue surface, as it improved slightly if a smooth plastic surface was used instead, but also on the difficulty found in accurately determining the focal point of the $\mathrm{HeNe}$ marker beam.

When the convergence angle of the two laser beams was increased by a factor of three, the focal point of the HeNe beam could be found more reliably, and at the same time the effective depth of focus for the YAG pulses was significantly reduced resulting in a more useful delivery system.

This system enabled the effects of displacing the target from the geometric focus, of lowering pulse energy, and of changing the nature of the target to be observed. Although these effects could have been predicted qualitatively, our system allowed precise measurements of the changes and corresponding effects to be made. It also showed that holes of the required morphology-diameter $100 \mu \mathrm{m}$ and depth sufficient to reach Schlemm's canal-could be created quite easily.

Similar in vitro studies are currently being pursued with fresh tissue rather than fixed being used, an even larger beam convergence angle, and a specially designed gonioglass, to approach the in vivo situation more closely. In this way we hope to gather systematically the data on which a clinical neodymium/YAG laser system and operating protocol can be soundly based.

This study was supported by the Rank Prize Fund (Grant No 7269).

\section{References}

1 Aron-Rosa D, Griesemann JC, Aron JJ. Applications ophtalmologiques des lasers neodymium YAG pulses-Ouvertures preoperatoires des cristallins avant implants et des cataractes secondaire derrière implants cristalliniens. J Fr Ophtalmol 1981; 4: 61-6.

2 Aron-Rosa D, Cohn HC, Aron T. Use of a pulsed neodymiumYAG laser (picosecond) in endocular surgery. In: Birngruber R, Gabel V-P, eds. Laser treatment and photocoagulation of the eye. Amsterdam: Junk, 1984.

3 Van der Zypen E, Bebie H, Fankhauser F. Morphological studies about the efficiency of laser beams upon the structures of the angle of the anterior chamber. Int Ophthalmol 1979; 1: 109-22.

4 Fankhauser F, Lortscher H, Van der Zypen E. Clinical studies on high and lower power laser radiation upon some structures of the anterior and posterior segments of the eye. Int Ophthalmol 1982; 5: $15-32$.

5 Fankhauser F, Roussel P, Steffen J, et al. Clinical studies upon some structures of the anterior segment of the eye. Int Ophthalmol $1981 ; 3: 129-39$.

6 Van der Zypen E, Fankhauser F. The ultrastructural features of laser trabeculopuncture and cyclodialysis. Ophthalmologica 1979; 179: 189-200.

7 Van der Zypen E, Fankhauser F. Lasers in the treatment of chronic simple glaucoma. Trans Ophthalmol Soc UK 1982; 102: 147-53.

8 Bloembergen $\mathrm{N}$. Laser-induced electric breakdown in solids. IEEE J Quant Electr 1974; QE10: 375-86. 